\title{
Clinicopathological significance of synchronous carcinoma in colorectal cancer
}

Alfred King-Yin Lam¹ ${ }^{1}$, M.B.B.S, M.D., Ph.D., F.R.C.P.A.

Robert Carmichael ${ }^{2}$, M.B.B.S

Petra Gertraud Buettner ${ }^{3}$, MSc, PhD

Vinod Gopalan ${ }^{1}$, B.H.M.S, M.Sc

Yik-Hong $\mathrm{Ho}^{3}$, M.B.B.S., M.D., F.R.A.C.S.

Simon $\mathrm{Siu}^{2}$, M.B.B.S, F.R.A.C.S.

${ }^{1}$ Division of Pathology (School of Medicine), Griffith Institute of Health and Medical

Research, Griffith University, Gold Coast, Queensland, Australia

${ }^{2}$ Divsion of Colorectal Surgery, Royal Brisbane and Women’s Hospital, Brisbane,

Queensland, Australia

${ }^{3}$ Division of Surgery (School of Medicine) and North Queensland Centre for Cancer

Research (Australian Institute of Tropical Medicine), James Cook University,

Townsville, Queensland, Australia

Correspondence and/or reprint requests:

Professor Alfred Lam

Foundation Chair Professor and Head of Pathology

Griffith Medical School

Griffith University Gold Coast Campus

Gold Coast QLD 4222

Australia

Phone+61 7 56780718; Fax +61 7 56780708; E-mail: a.lam@griffith.edu.au 


\section{SUMMARY FOR TABLE OF CONTENT}

When compared with solitary colorectal carcinoma, synchronous colorectal

carcinoma was more often noted in males, with co-existing FAP and in proximal

location. The 5-year survival rate of patients with synchronous colorectal carcinoma

was similar to those with solitary colorectal carcinoma.

KEYWORDS: synchronous, colorectal cancer, adenocarcinoma. 


\section{ABSTRACT}

BACKGROUND: Synchronous colorectal carcinoma has seldom been studied in large series. The study was designed to examine the significance of colorectal synchronous carcinoma in a large cohort of patients.

METHODS: The clinicopathological features of 102 patients with synchronous colorectal carcinoma were compared with 1793 patients with solitary colorectal carcinoma.

RESULTS: The prevalence of synchronous colorectal carcinoma was 3.6\%. In these patients, 4\% had familial adenomatous polyposis (FAP), 6\% had hyperplastic polyposis and $2 \%$ had ulcerative colitis. The index carcinoma was more likely to have higher histological grade and T stage than other carcinoma(s) in the same patient. When compared with solitary colorectal carcinoma, synchronous colorectal carcinoma was more often noted in males, with co-existing FAP and in proximal location. The 5-year survival rate of patients with synchronous colorectal carcinoma was $53 \%$ and was similar to those with solitary colorectal carcinoma.

CONCLUSIONS: We examined the clinicopathological features of patients with synchronous colorectal carcinomas in a large cohort of patients. Attention to these features was important for better management of this group of cancer.

Keywords: synchronous, multiple, colorectal, cancer, adenocarcinoma. 


\section{INTRODUCTION}

Synchronous colorectal carcinomas refer to more than one primary colorectal carcinoma detected in a single patient at time of initial presentation. It is likely that these carcinomas arise from a field effect of combined environmental and genetic origin. Recent advances in molecular biology have provided evidence that chromosomal instability (CIN), microsatellite instability (MSI) and gene methylation accounted for various predisposing lesions or factors for synchronous cancer [1]. CIN positive status (leading to inactivation of tumor suppressor genes) accounts for approximately $60 \%$ of synchronous cancer. This group of synchronous cancer includes ulcerative colitis-related cancer and familial adenomatous polyposis-related cancer. The other synchronous cancers are often MSI-high. Those cancers that are MSI-high and negative for CpG island methylation often present in the proximal colon. They account for approximately $10 \%$ of synchronous cancer. The other synchronous cancers that are positive for CpG island methylation (which leads to silencing of tumor suppressor gene) comprise approximately $30 \%$ of synchronous cancer. The category includes synchronous cancer associated with hyperplastic polyposis.

Although synchronous colorectal carcinoma is recognized as a significant entity on a clinical and molecular level, its clinical, pathological features and prognosis are still controversial. The difficulties of assessing the characteristics of synchronous colorectal carcinoma may be partly related to its relative low prevalence. A recent big population-based study on 15,562 colorectal cancers documented that $3.8 \%$ of newly diagnosed colorectal cancers had synchronous colorectal cancers [2]. This study defined the synchronous colorectal cancers has to be diagnosed simultaneously or within 6 months of the diagnosis of the index cancer. In some 
clinical series on synchronous colorectal cancers, they included cancers diagnosed at a much longer time interval. Some series also included metachronous colorectal cancers in the analysis [3]. It is worth noting that many clinical series were based on the analysis of less than half a hundred patients [3]. On the other hand, though the large population-based studies presented good demographic data and correlation statistics, these studies lack of detail features of the disease entity.

The objectives of this study were to investigate, in depth, the various clinicopathological features of patients with synchronous colorectal carcinoma in a large cohort of patients with long term follow-up data. The findings were also compared with those patients with solitary colorectal cancer. It is anticipated that the picture drawn from this study can bridge the gap of information provided by the larger population-based studies and smaller clinical series. 


\section{METHOD}

Patients with synchronous carcinoma in the colorectum were collected from the main tertiary hospital (Royal Brisbane and Women’s Hospital) and other regional hospitals in Queensland, Australia. The characteristics of these synchronous colorectal carcinomas were compared with those of other 1793 consecutive solitary colorectal carcinomas presenting to the Colorectal Surgical Unit of the Royal Brisbane Hospital between 1984 and 2004. The study data was collected in a computerized database and its accuracy ensured through employment of a trained clinical nurse for data entry and random selection of patients on database for rechecking their data against the original data sources by the staff and authors.

The main sources of the demographical, clinical, surgical and postoperative data were patients' medical records including specialist and/or general practitioner referral letters, pre-operative assessment records, admission notes, operative and anesthetic records, inpatient progress notes and discharge summaries in addition to direct consultation with patients at the time of presentation or at a follow up visit. The pathological information was obtained from the pathology reports and checked by the authors (AKL and RC). In addition to regular follow up, further information about survival of patients was obtained by accessing the state's main registry of death (Queensland Registry of Births, Deaths and Marriages) database. All the colorectal carcinomas were re-classified according to the recent AJCC TNM staging system by the authors (AKL and RC) [4].

Synchronous colorectal adenocarcinoma was defined as more than one primary adenocarcinoma in the colorectum at the time of resection or within 6 months. Metachronous colorectal adenocarcinoma was classified as occurrence of another primary colorectal adenocarcinoma more than 6 months after the detection of 
the primary colorectal adenocarcinoma. Only adenocarcinoma (including conventional adenocarcinoma or mucinous adenocarcinoma) was included in the study. Adenoma and other type of malignancies were noted included. In our series, each of the patients had the synchronous colorectal carcinomas detected at the same time. The pathological features of every synchronous colorectal cancer were studied. For every patient, the index carcinoma (labeled as S1) was defined as the one with most advanced $\mathrm{T}$ staging. If the carcinomas were of the same T-stage, the one with the largest size was labeled as S1. The second largest carcinoma in the same patient was labeled as S2. The histological grade (differentiation), histological subtype, size, location and T-staging of each of the synchronous cancers in every patient was analyzed and entered into database for analysis.

Management was by a pre-agreed standardized multidisciplinary protocol. The use of post-operative adjuvant therapy was based on the pathological stages and not on the presence of synchronous cancer. The follow-up period was defined as the interval between the date of surgery for colorectal carcinoma and the date of death or closing date of the study. The actuarial survival rate of the patients was calculated from the date of surgical resection of the colorectal carcinomas to the date of death or last follow-up. Only cancer-related death was counted as end point in the statistical analysis. The disease-free survival was calculated from the time of surgery to the date of first recurrence of the cancer.

\section{$\underline{\text { Statistical analysis }}$}

Fisher's exact test or likelihood ratio was used for categorical variables. Student t-test with Yates correction was used for continuous variables. The significance of various parameters on survival was analyzed by the Kaplan-Meier 
method with log-rank test and multivariate Cox’s regression. Significance level was taken at $\mathrm{p}<0.05$. All statistical tests were performed with the program, Statistical Package for Social Sciences (SPSS version 17.0, Chicago, IL). 


\section{RESULT}

\section{Clinicopathological features of synchronous carcinomas}

One hundred and two patients (69 men; 33 women) with synchronous colorectal carcinoma were noted. Of these, 68 patients were from a single institution with 1868 patients having colorectal carcinoma. Thus, the overall prevalence of the synchronous colorectal carcinoma was 3.6\%. The median age of the 102 patients was 70 (range, 24 to 92$)$ years. In these patients, $4 \%(n=4)$ had familial adenomatous polyposis (FAP), 6\% $(n=6)$ had hyperplastic polyposis and $2 \%(n=2)$ had ulcerative colitis. Obstructive symptoms were noted in $10 \%(\mathrm{n}=10)$ of the patients.

Complete colonoscopy was used in pre-operatively in 84\% (86 of 102) of patients and sigmoidoscopy was used in $5 \%(n=5)$. No pre-operative endoscopy was performed in $11 \%(n=11)$ of the patients as the resection of the cancers was performed in emergency. In the 86 patients with complete colonoscopy used per-operatively, 19\% ( $n=16)$ missed the synchronous lesion. The missed synchronous lesion was usually small in size or very close to the main cancer.

All the patients had single operation for the synchronous colorectal cancers. In $6 \%(n=6)$ of the patients, right hemi-colectomy and anterior resection were performed at the same time. The other operations comprised anterior resection (29\%, $n=30)$, total colectomy $(25 \%, n=25)$, right hemi-colectomy $(12 \%, n=12)$, left hemicolectomy $(8 \%, n=8)$, subtotal colectomy $(8 \%, n=8)$, extended right hemi-colectomy $(6 \%, n=6)$, abdominal- perineal resection $(5 \%, n=5)$ and Hartmann's procedure $(2 \%$, $n=2)$.

Majority of the patients ( $\mathrm{n}=86$; 84\%) had 2 synchronous colorectal carcinomas. In 12 patients (12\%), 3 synchronous colorectal carcinomas were noted. Other than these, 3 patients (3\%) had 4 synchronous colorectal carcinomas and 1 had 
6 synchronous carcinomas. Overall, a total of 226 colorectal cancers were noted in these 102 patients. The mean diameter of the carcinomas was 38mm (range, 2 to 188 $\mathrm{mm})$. The carcinomas were located in caecum in $12 \%(\mathrm{n}=28)$, ascending colon in $15 \%(n=34)$, hepatic flexure in $7 \%(n=15)$, transverse colon in $10 \%(n=23)$, splenic flexure in $4 \%(n=9)$, descending colon in 6\% $(n=14)$, sigmoid colon in $25 \%(n=56)$ and rectum in $21 \%(n=47)$.

The carcinomas were TNM stage I in $4 \%(n=4)$, stage II in 35\% (n=36), stage III in $37 \%(n=38)$ and stage 4 in 24\% ( $=24)$. The mean follow-up period for the 102 patients was 48 months. Twenty-nine percent $(n=30)$ of the patients died of causes related to the colorectal carcinoma in the study period. The 5 year cancer survival rate and disease free survival rate of the whole group was 53\% and 52\% respectively. By Kaplan-Meier log-rank survival and Cox-multivariate analysis, survival of the patients with colorectal cancer was only associated with the TNM stages of the colorectal carcinomas $(\mathrm{p}=0.0001)$. Two patients $(2 \%)$ had metachronous colorectal carcinoma presenting at 82 months and 106 months respectively after the resection.

\section{Comparison between the synchronous cancers}

The difference in features between the largest cancer (S1) and the second largest cancer (S2) were shown in Table 1. The site distribution of S1 was similar to those of S2. In general, S1 was usually larger in dimension than S2 (mean $=52 \mathrm{~mm}$ versus $28 \mathrm{~mm}, \mathrm{p}=0.0001)$. Majority of the S1 (91\%) were of advanced T stages (T3 or T4). In contrast, over half (57\%) of S2 was of less advanced T stages (T1 or T2). The difference was statistically significant ( $p=0.0001)$. In 64\% $(n=65)$ of the patients, S1 was of higher T stage than S2 and the other was of identical T stage. 
With respect to pathology, $73 \%(n=74)$ had both S1 and S2 of the same histological grade. Other than this, S1 had higher histological grade than S2 in 23\% $(n=24)$ of patients. In 4\% ( $n=4)$ of patients, S2 had higher histological grade than S1. Overall, S1 was more likely to be poorly-differentiated than S2 ( $\mathrm{p}=0.002)$.

Mucinous adenocarcinoma was noted in $18 \%$ of S1 whereas the subtype was noted in $8 \%$ of S2. However, the difference did not reach statistical significant level $(\mathrm{p}=0.057)$. In addition, $80 \%(\mathrm{n}=82)$ of patients had both $\mathrm{S} 1$ and S2 of the same histological subtypes. Of these, 3 had both S1 and S2 of mucinous subtype.

The colorectum was dividing into 5 segments for the analysis of location of the synchronous cancers. These comprised (1) caecum, (2) ascending colon, (3) transverse colon + splenic flexure + hepatic flexure, (4) sigmoid colon and (5) rectum. Of the synchronous cancers, 38 patients (37\%) had the tumors noted in the same colorectal segment. Amongst these, these carcinomas were most often noted in either sigmoid colon $(n=13)$ or rectum $(n=10)$. The remaining 64 patients had the carcinomas either in adjacent segments $(n=31)$, separated by 1 segment $(n=14)$, separated by 2 segments $(n=12)$, separated by 3 segments $(n=5)$ and separated by 4 segments $(n=2)$.

\section{$\underline{\text { Synchronous versus solitary colorectal cancer }}$}

The difference between the 102 synchronous colorectal carcinomas and the 1793 solitary colorectal carcinomas were shown in Table 2.

When compared with solitary colorectal carcinoma, synchronous colorectal carcinoma was more often noted in males $(\mathrm{p}=0.006)$. Also, synchronous carcinoma was more likely to be of higher histological grade and more advanced TNM stages ( $p=0.039$ and 0.002 respectively). Synchronous carcinoma, when compared with 
solitary carcinoma, was more often seen in proximal colon especially the ascending colon ( $\mathrm{p}=0.0001)$. In patients with solitary colorectal carcinoma, the most common site of the carcinoma was in the rectum (33\%) whereas in patients with synchronous carcinoma, the most common site was in sigmoid colon (25\%). As a whole, synchronous carcinoma was more often noted in proximal colon than solitary carcinoma (44\% versus $34 \%, \mathrm{p}=0.003)$.

Co-existing FAP was more often seen in patients with synchronous colorectal cancer than solitary colorectal carcinoma $(\mathrm{p}=0.0001)$. Ulcerative colitis was also more common noted in patients with solitary colorectal carcinoma. However, the difference was marginally significant $(\mathrm{p}=0.051)$. The two categories of colorectal cancer did not differ from each other in patients' age, size of cancer, presence of obstructive symptoms, presence of metachronous colorectal cancer, pre-operative serum carcinoembryonic antigen (CEA) level, histological subtype and cancer recurrent rates $(\mathrm{p}>0.05)$.

The two groups of carcinoma did not significantly differ from each other on either disease free survival or cancer-related death survival. On multi-variant survival analysis, the difference between the 2 groups in cancer-related death was marginally insignificant $(\mathrm{p}=0.058)$. For stages 3 cancers, synchronous colorectal cancers appeared to do slightly better in survival rate. The 5-year survival rate for stage III synchronous colorectal cancers was $80 \%$ whereas that for stage III solitary colorectal cancer was $46 \%(\mathrm{p}=0.05)$. 


\section{DISCUSSION}

Predisposing lesions like ulcerative colitis and FAP are well known in the pathogenesis of synchronous colorectal carcinoma. Patients with FAP or ulcerative colitis were noted to be having higher frequencies of synchronous colorectal carcinoma than in general population [3]. A study performed by Greenstein et al reported that synchronous cancer accounted for $2.5 \%$ for de novo colorectal cancer, $18 \%$ for ulcerative colitis-related cancer and 21\% for FAP-related cancer [3]. Also, in the recent literature, synchronous colorectal cancer was reported to be a common feature of hyperplastic polyposis [5]. However, the relative prevalence of these predisposing factors in the synchronous colorectal cancer was rarely documented. In this study, we noted that ulcerative colitis, FAP and hyperplastic polyposis accounted for $2 \%, 4 \%$ and $2 \%$ of synchronous colorectal carcinoma. Also, FAP and ulcerative colitis were more commonly found in the patients with colorectal synchronous carcinoma than solitary colorectal carcinoma. Overall, ulcerative colitis, FAP and hyperplastic polyposis are documented to be important contributing factors in synchronous colorectal carcinoma. Altogether, they accounted for $10 \%$ of synchronous colorectal cancer in the current study.

In the literature, the prevalence of synchronous colorectal carcinoma ranged from approximately 1 to $8 \%$ [3,6]. Nevertheless, many of the series are small and the figures are unlikely to be representative. In series analyzing more than 50 synchronous colorectal carcinomas, the prevalence of the entity was 2 to $6 \%[7,8]$. In recent decade, the only large study on synchronous colorectal cancer was a population based study by Latourneire et al in France in which the entity was found to be accounted for 3.8\% $(n=596)$ of 15,562 colorectal cancer [2]. The present study based on surgical series analyzing 102 synchronous colorectal cancers is the largest 
hospital based series in the current few decades. The prevalence found in this study was 3.6\% and similar to those obtained by Latourneire et al [2]. Thus, the true prevalence of synchronous colorectal synchronous cancer is likely to be slightly less than $4 \%$.

Discrepancies have been reported of the implications of demographic factors such as gender and age at diagnosis on the frequencies of colorectal synchronous cancer. In most studies, synchronous colorectal cancers were more frequent in men $[3,9]$. In the present study, being male was also a risk factor for having synchronous colorectal carcinoma. Around two third (68\%) of the patients with synchronous colorectal cancer were men. There is no obvious reason for this male predominance for this entity. It is likely the genetic and environmental factors may contribute to gender difference in prevalence. With respect to age at diagnosis, there is no common consensus on its effect on the prevalence of synchronous colorectal cancer. Studies have reported a younger age of diagnosis, older age of diagnosis or similar age distribution between synchronous and solitary colorectal cancer $[2,14,15]$. In large series, like the present series and that by Latourniere et al, the age at diagnosis for solitary colorectal and synchronous colorectal carcinoma was similar [2].

The location of the synchronous cancers also varies with series and the relative incidence when compared to solitary cancer $[16,17]$. In our series, we noted that the most common site of occurrence of synchronous carcinoma was sigmoid colon (25\%) and the second commonest site was the rectum (21\%). This is similar to solitary colorectal carcinoma in which the most common site was rectum and the second commonest site was sigmoid colon. On the other hand, the relative frequencies in different portion of the colorectum were difference for synchronous and solitary colorectal cancers. When compared to solitary colorectal carcinoma, synchronous 
carcinoma appeared to be more often involving the proximal portion of the colon, in particular the ascending colon. These findings were in concurrence with those from other series and also in lines with the right side predominance effect of some genetic predisposing factors [17].

The pathological features of the synchronous colorectal cancers were seldom analyzed. In general, it was reported that synchronous colorectal cancers often occur in the same or adjacent segment of the large intestine $[2,9]$. The other cancer(s) in the patients with synchronous colorectal carcinomas were usually smaller, lower pathological grade and T staging than the index cancer. In our study, we noted that the same tendency for the synchronous colorectal cancers. We also detected that mucinous adenocarcinoma was slightly more common in synchronous colorectal carcinoma. In 3 of the 82 patients with identical histological subtypes between the synchronous carcinomas (index and other cancers in the same patient), both the index and synchronous carcinomas were mucinous adenocarcinoma.

In this study, we did not find any correlation between the presence of obstructive symptoms or pre-operative serum CEA level and the presence of synchronous colorectal cancer. In addition, we noted that $68 \%$ of the patients had synchronous cancers in the same or adjacent segment of the colorectum. Only $19 \%$ $(n=19)$ of the cancers were separated by 2 or more anatomical segments (which means one cancer in proximal colon and one in distal colorectum). Thus, clinical features were not helpful in the detection of the cancers. On the other hand, the synchronous carcinomas were often close to each other which may make clinical and pathological detection easier.

The prognosis of patients with synchronous carcinoma has been documented to be better, the same or worse than those with solitary colorectal carcinoma $[2,18$, 
19]. The variation is likely due to sample size, length of follow-up etc and needs to be interpreted with caution. In our study on a large cohort of patients with long-term follow-up data, we noted no difference in survival rates, cancer recurrent rates, incidence of metachronous colorectal cancer between patients with solitary and synchronous colorectal carcinoma. The survival of the patients with synchronous carcinoma only depended on the pathological staging of the index cancer. Also, in stage 3 cancers (the largest subgroup of colorectal cancer), we noted a marginally better survival rate in synchronous colorectal carcinoma than solitary colorectal cancer. The reason for the better survival rates was unknown but may be related to the higher susceptibility to adjuvant therapy for stage 3 synchronous cancers as the cancers are more genetic unstable. 


\section{REFERENCES}

1. Leggett BA, Worthley DL. Synchronous colorectal cancer: not just bad luck? Gastroenterology, 2009;137:1559-1562.

2. Latournerie M, Jooste V, Cottet V, Lepage C, Faivre J, Bouvier AM. Epidemiology and prognosis of synchronous colorectal cancers. Br J Surg, 2008;95:1528-1533.

3. Greenstein AJ, Slater G, Heimann TM, Sachar DB, Aufses AH Jr. A comparison of multiple synchronous colorectal cancer in ulcerative colitis, familial polyposis coli, and de novo cancer. Ann Surg, 1986;203:123-128.

4. Oh HS, Chung HJ, Kim HK, Choi JS. Differences in overall survival when colorectal cancer patients are stratified into new TNM Staging strategy. Cancer Res Treat, 2007;39:61-64.

5. Yeoman A, Young J, Arnold J, Jass J, Parry S. Hyperplastic polyposis in the New Zealand population: a condition associated with increased colorectal cancer risk and European ancestry. N Z Med J, 2007;120:U2827.

6. Wang HZ, Huang XF, Wang Y, Ji JF, Gu J. Clinical features, diagnosis, treatment and prognosis of multiple primary colorectal carcinoma. World J Gastroenterol, 2004;10:2136-2139.

7. Welch JP. Multiple colorectal tumors. An appraisal of natural history and therapeutic options. Am J Surg, 1981;142:274-280.

8. Lasser A. Synchronous primary adenocarcinomas of the colon and rectum. Dis Colon Rectum, 1978;21:20-22.

9. Oya M, Takahashi S, Okuyama T, Yamaguchi M, Ueda Y. Synchronous colorectal carcinoma: clinico-pathological features and prognosis. Jpn J Clin Oncol, 2003;33:38-43.

14: Ueno M, Muto T, Oya M, Ota H, Azekura K, Yamaguchi T. Multiple primary cancer: an experience at the Cancer Institute Hospital with special reference to colorectal cancer. Int J Clin Oncol, 2003;8:162-167.

15. Dykes SL, Qui H, Rothenberger DA, García-Aguilar J. Evidence of a preferred molecular pathway in patients with synchronous colorectal cancer. Cancer, 2003;98:48-54.

16. Ikeda Y, Saku M, Kawanaka H, Muranaka T, Takeshita M, Watanabe J, Yoshida $\mathrm{K}$, Sugimachi K. Distribution of synchronous and metachronous multiple colorectal cancers. Hepatogastroenterology, 2004;51:443-446.

17. Kimura T, Iwagaki H, Fuchimoto S, Hizuta A, Orita K. Synchronous colorectal carcinomas. Hepatogastroenterology, 1994;41:409-412. 
18. Nosho K, Kure S, Irahara N, Shima K, Baba Y, Spiegelman D, Meyerhardt JA, Giovannucci EL, Fuchs CS, Ogino S. A prospective cohort study shows unique epigenetic, genetic, and prognostic features of synchronous colorectal cancers. Gastroenterology, 2009;137:1609-1620.

19. Wagner HE, Barbier PA, Luder PJ, Niederhäuser U. Prognosis in synchronous colorectal carcinomas. Z Gastroenterol, 1988;26:117-120. 
Table 1.

Comparison between the largest (S1) and second largest cancer (S2) in 102 synchronous colorectal cancers

S1 S2 p-value

\section{Grade}

Well

Moderate

$2(2 \%)$

$72(71 \%)$

$4(4 \%)$

0.002

Poor

$28(27 \%)$

$89(87 \%)$

$9(9 \%)$

Histological subtype

Adenocarcinoma

$84(82 \%)$

$94(92 \%)$

0.057

Mucinous adenocarcinoma

$18(18 \%)$

8 (8\%)

\section{Location}

Caecum

Ascending colon

$15(12 \%)$

$13(15 \%)$

$10(10 \%)$

0.478

Hepatic flexure

$7(7 \%)$

$15(15 \%)$

Transverse colon

$13(10 \%)$

$5(5 \%)$

$7(7 \%)$

$5(4 \%)$

$4(4 \%)$

Descending colon

$5(6 \%)$

$9(9 \%)$

$21(25 \%)$

$21(20 \%)$

$23(21 \%)$

$21(20 \%)$

Rectum

28

0.0001

Mean maximum dimension 52

(in mm)

\section{T staging}

I

II

$1(1 \%)$

$8(8 \%)$

$33(32 \%)$

0.0001

III

$72(71 \%)$

$25(25 \%)$

$41(40 \%)$

IV

$21(20 \%)$

$3(3 \%)$ 
Table 2. Comparison between synchronous and solitary colorectal cancer

\begin{tabular}{|c|c|c|}
\hline & Synchronous & Solitary \\
\hline Total number & $\begin{array}{l}102 \text { patients/ } \\
226 \text { cancers }\end{array}$ & 1793 patients \\
\hline
\end{tabular}

Gender

Male

$69(68 \%)$

$959(53 \%)$

0.003

Female

$33(32 \%)$

$834(47 \%)$

Age (years)

68

67

0.207

Grade

Well

$2(2 \%)$

$74(73 \%)$

Moderate

$26(25 \%)$

$100(6 \%)$

0.039

Poor

$1386(77 \%)$

$307(17 \%)$

Mean maximum dimension 52

(in $\mathrm{mm}$ )

50

0.641

\section{Histological subtype}

Adenocarcinoma

$85(83 \%)$

$1518(85 \%)$

0.674

Mucinous adenocarcinoma

$17(17 \%)$

$275(15 \%)$

\section{Location}

Caecum

$28(12 \%)$

34 (15\%)

$15(7 \%)$

$23(10 \%)$

$9(4 \%)$

$14(6 \%)$

$56(25 \%)$

47 (21\%)

$276(15 \%)$

0.0001

Hepatic flexure

Transverse colon

Splenic flexure

Descending colon

Sigmoid colon

$125(7 \%)$

$77(4 \%)$

$134(8 \%)$

$68(4 \%)$

$67(4 \%)$

$446(25 \%)$

600 (33\%)

\section{TNM staging}

I

$4(4 \%)$

$36(35 \%)$

$280(16 \%)$

0.002

II

$38(37 \%)$

$658(37 \%)$

439 (24\%)

IV

24 (24\%)

$416(23 \%)$

Associated FAP

$4(4 \%)$

$3(0.2 \%)$

0.0001

Associated ulcerative colitis 2 (2\%)

$5(0.3 \%)$

0.051

Metachronous cancer

$2(2 \%)$

$39(2 \%)$

1 